\title{
A Spatio-Temporal Approach to Selective Data Dissemination in Mobile Peer-to-peer Networks ${ }^{1}$
}

\author{
Yan Luo \\ Department of Computer Science \\ University of Illinois at Chicago \\ Chicago, IL, USA \\ yluo@cs.uic.edu
}

\author{
Ouri Wolfson \\ Pirouette Software Consulting and \\ University of Illinois at Chicago \\ Chicago, IL, USA \\ wolfson@cs.uic.edu
}

\author{
Bo Xu \\ Department of Computer Science \\ University of Illinois at Chicago \\ Chicago, IL, USA \\ boxu@cs.uic.edu
}

\begin{abstract}
We examine data dissemination in mobile peer-to-peer networks, where moving objects communicate with each other via short-range wireless technologies such as IEEE 802.11 or Bluetooth. Given the memory and bandwidth/energy constraints at moving objects, the ideal mobile peer-to-peer dissemination method is for each moving object to store and transmit only the reports that are new to other objects encountered in the future. However, in practice no object can know the states of all the other objects due to the distributed and dynamic nature of mobile peer-to-peer networks. Thus, predicting the novelty probability of a report is important for efficient data dissemination in mobile peer-to-peer networks. In this paper we propose a decentralized spatio-temporal approach to selective data dissemination. In this approach, the novelty probability of a report is estimated based on both spatial and temporal attributes (AGE and DISTANCE) of the report and each moving object only stores and transmits the reports with the highest novelty probabilities. We study different strategies of using novelty factors to compute the novelty probability. Extensive experiments are conducted to test and analyze the performance of different strategies. The experimental results determine the best strategy and demonstrate its superiority against existing mobile peer-topeer methods.
\end{abstract}

\section{INTRODUCTION}

A mobile peer-to-peer network is a set of moving objects that communicate with each other via unregulated, short-range wireless technologies such as IEEE 802.11 or Bluetooth. No fixed infrastructure is assumed or relied upon. With devices such as mobile phones, PDAs, digital cameras, and supporting wireless networks, mobile peer-to-peer computing will permeate our daily life via both non-critical civilian applications and critical emergency-response operations. Mobile peer-to-peer networks can be used for people to share information and resources in a peer-to-peer manner, such as exchanging personal cards, photos, music, and even videos. Mobile peer-to-peer networks can be also used for monitoring or managing the real-time traffic, and for assisting travelers in efficiently using transportation systems, e.g., finding parking slots or requesting ride-sharing. Another application scenario is that when driving on a jammed highway one can know the average speed one or two miles ahead by sending a query and receiving the answer via multi-hop transmissions relayed by intermediate vehicles. Another civilian application in which mobile peer-to-peer networks are useful is the directed

\footnotetext{
${ }^{1}$ Research supported by NASA Award NNA06AA25C, and NSF Grants OII-0611017, DGE-0549489, 0513736, and 0326284.
}

advertisement in mobile commerce or tourism; for example, disseminating timetables of local transportation or menus of a restaurant. In emergency response situations, mobile peer-topeer networks can be used to propagate the request-for-help messages through pedestrians and vehicles quickly, especially after the infrastructure has failed.

In mobile peer-to-peer networks, since the ranges of 802.11 or Bluetooth are not sufficient to reach all interested moving objects, data dissemination is done by transitive multi-hop transmission. Many traditional data dissemination paradigms assume that a data-item (from now on called report) can be transferred immediately after it is received. However, when the communication network is sparsely connected, the moving objects may have to store the reports, and later (as new neighbors are discovered) transfer these reports. Moreover, due to the limited memory and bandwidth/energy, moving objects can only store and transmit a limited number of reports during each transmission. In other words, reports need to be prioritized in order to accommodate the limited memory and bandwidth/energy.

Consider the flooding scenario, in which each report is to be disseminated to all the moving objects. In this case, an important factor that determines the priority of a report is its current novelty probability, i.e. its probability of being new (i.e. previously unknown) to an arbitrary moving object encountered in the future. The reason is that the transmission of reports that are already known wastes bandwidth, memory, and energy. Thus in this paper reports are ranked based on their current novelty probability.

Under uniformity assumptions, the novelty probability of a report $R$ at a particular time is simply the ratio between the total number of moving objects that have received $R$, and the total number of moving objects in the system. Unfortunately, a parametric formula giving the novelty probability is beyond the state of the $\mathrm{art}^{2}$. In this paper we propose a novelty prediction approach based on the intuition that the novelty probability of $R$ depends on some inherent attributes of $R$, which are called the novelty factors. For example, a later generated report has a higher novelty probability than an earlier generated one because the earlier generated one has probably been received by many objects. Thus, the age of a report is a novelty factor from the temporal perspective. Similarly, the distance between the location at which the report is generated and the location at

${ }^{2}$ Results from epidemiology and random graphs may be useful in deriving such a formula, but they can't be applied directly. 
which the novelty probability is computed is a novelty factor from the spatial perspective. We develop and compare several strategies of estimating novelty probability using AGE and DISTANCE. For example, the estimation may be based on either AGE or DISTANCE, or a combination of the two. Observe that our approach is stateless, in the sense that neither routing information nor global information such as the knowledge of the destination nodes is required.

To test and analyze the performance of the proposed spatiotemporal approach, we develop a software system to simulate data dissemination in mobile peer-to-peer networks. In the system we use the Random Way Points (RWP) mobility model and the new reports are randomly injected to a set of moving objects. We compare various strategies using a performance measure that integrates throughput and response-time. Extensive experiments are conducted to verify our intuitions for efficient data dissemination. The experimental results show that the combination of AGE and DISTANCE is the best strategy for a wide variety of mobile peer-to-peer network environments.

The rest of the paper is organized as follows. Section II formalizes the data dissemination problem and analyzes the challenges. Section III proposes the novelty factors and the spatio-temporal approach. Section IV evaluates the approach by simulations. Section V discusses relevant work. Section VI concludes the paper and discusses the future work.

\section{DATA DiSSEMINATION MODEL}

A mobile peer-to-peer network is a finite set of moving objects. We assume that each moving object knows its current location through some localization device, such as a GPS. Each moving object has a memory allocation and a bandwidth/energy allocation that are dedicated to data dissemination. Occasionally, a moving object $O$ produces a report $R$ having some report-id. For example, $R$ is a set of values simultaneously sensed by the moving object. In our model time is a sequence of discrete atomic time units, $1,2,3 \ldots \mathrm{n}$, and location is a two-dimensional point $(x, y)$. Formally report $R$ is a 4-tuple (report-id, $t(R), l(R)$, values) in which report-id is a global unique id, $t(R)$ is the time at which $R$ is produced, referred to as the production time of $R$, and $l(R)$ is the location at which $R$ is produced, referred to as the production location of $R$. The size is the same for all the reports.

Each moving object $O$ maintains a reports database whose size is $M_{i}$ reports. The reports database is maintained solely for the purpose of disseminating the reports to other moving objects. In addition, $O$ has a memory area called the buffer that is common to all the applications running on the moving object. We assume that the size of the buffer is at least $M_{i}$. Moving objects communicate with each other via short range wireless technologies such as IEEE 802.11 and Bluetooth. The transmission range is denoted by $d$. Thus, pair-wise communication can occur between two moving objects while the distance between them is smaller than $d$. When two moving objects A and B encounter each other (i.e., when the distance between them is smaller than $d$ ), they engage in a reports exchange. The number of reports exchanged depends on the bandwidth/energy of each moving object, the memory allocation of each, and the period of time for which they stay within transmission range.

\section{SPATIO-TEMPORAL APPROACH}

Given $t$ a point in time, the probability that a report $R$ at a moving object $O$ will be new to other objects encountered by $O$ after time $t$ is defined as the novelty probability of $R$ at $t$. Obviously, novelty probability can be calculated as the percentage of objects in the system that do not know $R$ at time $t$ However, the calculation cannot be done by any object since it does not know the state of other objects due to the distributed and dynamic nature of the system. Therefore, we propose a prediction approach based on the intuition that the novelty probability of $R$ depends on some inherent attributes of $R$, which are called the novelty factors. For example, from the temporal perspective, a newer report has higher novelty probability than an older report because the older report has probably been received by more objects. We formally define this temporal attribute as the $A G E$ of $R$, which is calculated to be the length of the time period starting from the production time $t(R)$ until the current time. On the other hand, from the spatial perspective, if a report has traveled a longer way from its production location it should have a lower novelty probability because during the travel it has probably been received by more objects. We formally define this spatial attribute as the DISTANCE of a report, which is calculated to be the distance between the current location and the production location $l(R)$.

Based on the above two novelty factors, we propose a distributed algorithm for data dissemination in mobile peer-topeer networks. The algorithm runs on each moving object $O$ and goes through three main phases, namely Initialization, Receiving, and Transmitting. These three phases are illustrated in Figure. 1 with shadowed boxes.

The Initialization phase is performed when $O$ starts to participate in data dissemination. In this phase, $O$ clears the reports database and resets a set of counters and timers, which are used for computing novelty probability. When $O$ encounters another object, the algorithm goes to the Receiving phase and the Transmitting phase. During these two phases, $O$ receives and transmits a number of reports. The Receiving phase at $O$ when it encounters a moving object $A$ is performed as follows:

1. Receives a set of reports from $A$ and temporally stores them in the buffer.

2. Discards from the buffer the reports that are already in the reports database of $O$.

3. Computes the novelty probabilities of the reports in the buffer and the reports in the database based on the selected novelty factors.

4. Sorts the reports in the buffer together with the reports in the database based on the selected ranking strategy, and saves the top $M_{O}$ reports (i.e., the $M_{O}$ reports with the highest novelty probabilities). Recall that $M_{O}$ is the size of the reports database of $O$. The ranking strategies will be discussed shortly. 
Similar to the Receiving phase, in the Transmitting phase, $O$ first computes the novelty probabilities of all reports stored in its reports database, and then sorts them based on the selected strategy, and finally transmits the top $b$ reports, where $b$ is determined by the bandwidth/energy allocation and the report size.

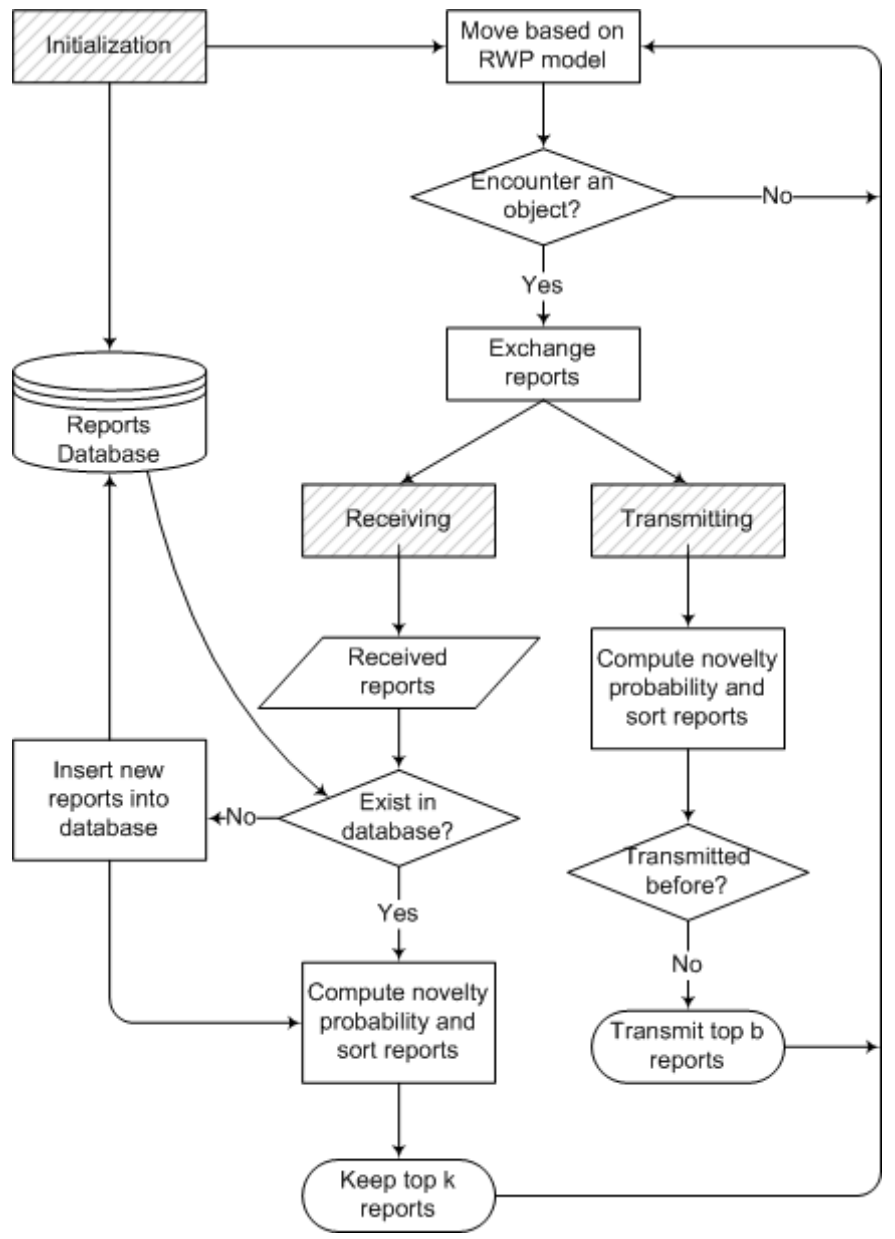

Figure. 1 The main algorithm running on every moving object

In the above algorithm, there are two phases, namely Receiving and Transmitting, where novelty probabilities are computed and reports are sorted. There are several alternatives depending on which novelty factors are used for ranking and in what order reports are sorted. As the first alternative, we can use a single novelty factor, either AGE or DISTANCE, to compute the novelty probability. For AGE, we only need to use the same sorting order in both Receiving and Transmitting, because the change of age will apply to all reports and the ranking of reports by using AGE will remain the same. However, for DISTANCE, since the location at which Receiving is performed and that at which Transmitting is performed may be different, the ranking of reports for using DISTANCE might be different. Thus, we have to compare different ranking strategies for using DISTANCE to compute the novelty probability. To validate our intuitions, we designed six different ranking strategies by using different novelty factors and different sorting orders, which are summarized in
Table.1. We will use the simulation experiments to determine which strategy is the best, and in which situations.

\begin{tabular}{|l|l|l|l|}
\hline Strategy & $\begin{array}{l}\text { Novelty } \\
\text { Factors }\end{array}$ & $\begin{array}{l}\text { Sorting for } \\
\text { Receiving }\end{array}$ & $\begin{array}{l}\text { Sorting for } \\
\text { Transmitting }\end{array}$ \\
\hline$\# 1$ & AGE & Ascending & Ascending \\
\hline$\# 2$ & AGE & Descending & Descending \\
\hline$\# 3$ & DISTANCE & Ascending & Ascending \\
\hline$\# 4$ & DISTANCE & Descending & Descending \\
\hline$\# 5$ & DISTANCE & Ascending & Descending \\
\hline$\# 6$ & DISTANCE & Descending & Ascending \\
\hline
\end{tabular}

Table. 1 Different strategies for updading novelty probability

Furthermore, we can combine the two novelty factors when computing novelty probability. In this paper we use the weighted sum of AGE and DISTNCE, i.e., Novelty Probability $=W_{1} * \mathrm{AGE}+W_{2} *$ DISTANCE, where $W_{1}$ and $W_{2}$ are weighting factors ranging from 0 to 1.0 . We refer to this strategy as Combination. The values of $W_{1}$ and $W_{2}$ can be tuned by simulation experiments for different environments.

\section{SIMULATION SYSTEM AND EXPERIMENTS}

In this section we evaluate the ranking strategies described in section III. To put the results in perspective, we compare these strategies with the spread mode of PeopleNet [18], in which reports are randomly selected for saving and transmission.

We developed a simulation system based on the formalized data dissemination problem and the Random Way Point (RWP) mobility model. The simulation environment area is a $0.5 \times 0.5$ mile square area. At the beginning of the simulation, a number of moving objects are introduced at some random points in the area, and then each of them moves at a constant speed along a straight line to another random point. There it pauses for a time interval, and then moves at a constant speed along a straight line to the next random point, and so on. At every second, a number of new reports are produced and this number is referred to as the producing rate. A produced report is instantaneously injected to a percentage of randomly selected moving objects, and this percentage is referred to as the injection rate. Each simulation runs for 600 seconds.

Each object has a database to store the reports and the maximum number of reports that can be stored is referred to as the database size. When two moving objects encounter each other, they follow the proposed algorithm and strategy to exchange the reports. During each exchange, a moving object can only transmit a fraction of its database due to the bandwidth/energy constraints. This fraction is referred to as the transmission fraction. For example, if the database size is 10 and the transmission fraction is 0.3 , then each moving object can transmit at most 3 reports during each transmission. The transmission fraction is the same for all the moving objects in one simulation but it may vary for different simulations. Each exchange is finished instantaneously. 
All the eight environmental parameters are listed in Table.2. For the Combination strategy we fine-tuned the weights to be $W_{1}=0.2$ and $W_{2}=0.8$ and used these values for all the experiments.

\begin{tabular}{|l|l|l|}
\hline Parameter & Description & Range \\
\hline$o b$ & number of moving objects & $10-100$ \\
\hline$s p$ & motion speed & $5-100$ miles/hour \\
\hline$p t$ & pause time & $10-100$ seconds \\
\hline$p r$ & producing rate & $1-10$ reports/second \\
\hline$i r$ & injection rate & $1 \%-80 \%$ \\
\hline$d b$ & database size & $1-100$ reports \\
\hline$t r$ & transmission range & $50-500$ meters \\
\hline$t f$ & transmission fraction & $0.1-1.0$ \\
\hline
\end{tabular}

Table. 2 Environmental parameters for the simulation system

As the performance measure for comparison of various ranking strategies we take a combination of throughput and response time; it averages the number of distinct reports received by a moving object within a certain time limit $c$. This is similar to the way an academic department is evaluated according to the percentage of its students that graduate within 4 years. More specifically, the measure takes the average number of reports with ages smaller than $c$ that are received by a moving object. This measure is called the response-time bounded throughput, or throughput, and $c$ is called the response-time bound. By varying the value of $c$, we evaluate the throughput of an algorithm for different response-time constraints.

To validate our intuitions and analyze the performance of different strategies, we first conducted the experiments in the default environment where all environmental parameters are equal to their default values listed in Table.3.

\begin{tabular}{|l|l|l|l|}
\hline Parameter & Default value & Parameter & Default value \\
\hline$o b$ & 75 & $i r$ & $1 \%$ \\
\hline$s p$ & 10 miles/hour & $d b$ & 10 reports \\
\hline$p t$ & 30 seconds & $t r$ & 150 meters \\
\hline$p r$ & 10 reports/sec. & $t f$ & 0.5 \\
\hline
\end{tabular}

Table. 3 Default values of environmental parameters

The experimental results are shown in Figure.2. First let us identify the best strategy for AGE-based ranking and that for DISTANCE-based ranking. From Figure. 2 it can be seen that when only AGE is used for novelty ranking, strategy \#1 is better than \#2. This is not surprising; younger reports have higher novelty probability since they have not been known by many moving objects. When only DISTANCE is used for novelty ranking, strategy \#5 is better than \#3, \#4, and \#6. This is a little bit difficult to understand and can be explained as follows. When a moving object receives some new reports and has to remove some ones due to the limited memory, it should keep the reports with shorter DISTANCE, since these reports have not been widely spread and therefore they have higher novelty probabilities. On the other hand, when a moving object transmits some reports to other moving objects, it should transmit the reports with longer DISTANCE, since longer
DISTANCE means that the current location is farther away from the production location of the report so that the report is more likely to be new to other moving objects around the current location.

The simulations with other parameter configurations point to the same conclusion, namely, \#1 is the best strategy for AGE-based ranking and \#5 is the best strategy for DISTANCEbased ranking. Thus, in the rest of this section, we only present the results of \#1 and \#5 for AGE-based ranking and DISTANCE-based ranking, respectively, with the results of \#2, \#3, \#4, and \#6 omitted.

Furthermore, observe that (i) the Combination strategy is better than all the other strategies; (ii) Combination, \#1 and \#5 are better than PeopleNet.

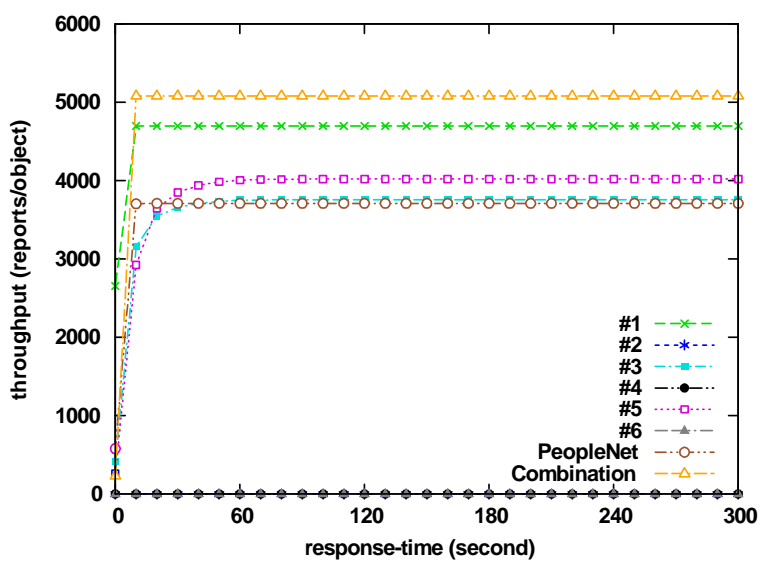

Figure. 2 Experimental results of different strategies with response-time

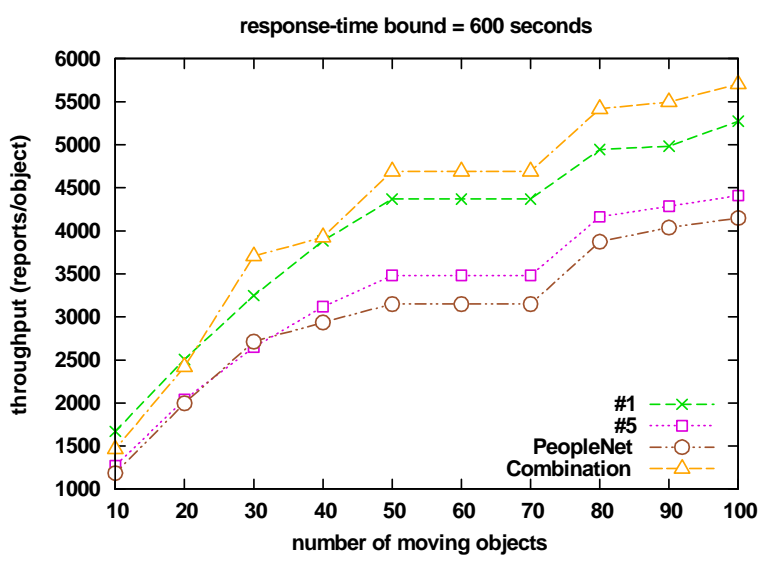

Figure. 3 Experimental results of varying object number

Figures 4, 5, and 6 compare the performance in different mobile environments in terms of object density, mobility, and bandwidth/energy allocation, with the response-time bound fixed to be 600 seconds. In each figure, we varied the value of one parameter with the values of all the other parameters fixed. For example, when we analyzed the object density, we kept all parameters fixed but only varied the number of moving objects $(o b)$ from 10 to 100 and recorded the performance of different strategies in Figure.3. From Figure. 3 it can be seen that the performance of all strategies increases with increasing $o b$, and 
Combination $>\# 1>\# 5>$ PeopleNet. Other comparison experimental results are illustrated in Figure.4 (varying the motion speed), Figure.5 (varying the transmission range), and Figure.6 (varying the transmission fraction) respectively. These experimental results show that the Combination strategy is always better than other strategies. This verifies that the knowledge from both spatial and temporal aspects produces better results. The performance of PeopleNet is almost always the worst and the performance of AGE is always better than DISTANCE.

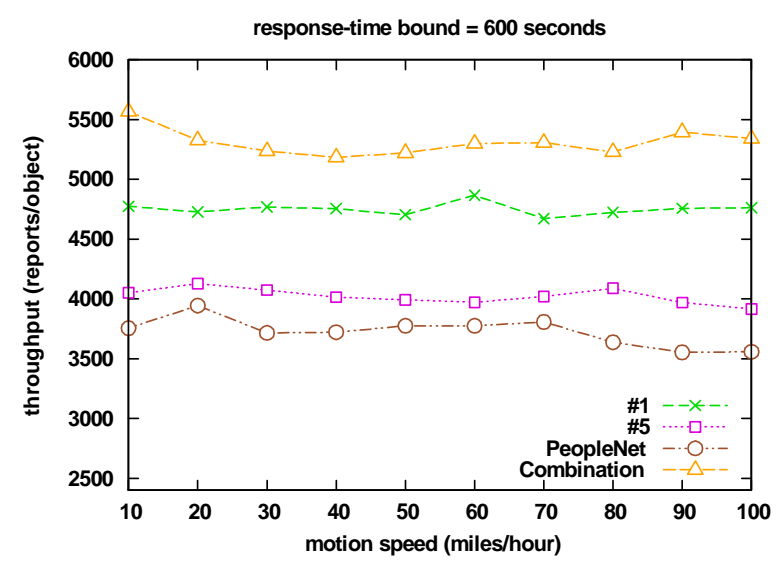

Figure. 4 Experimental results of varying speed

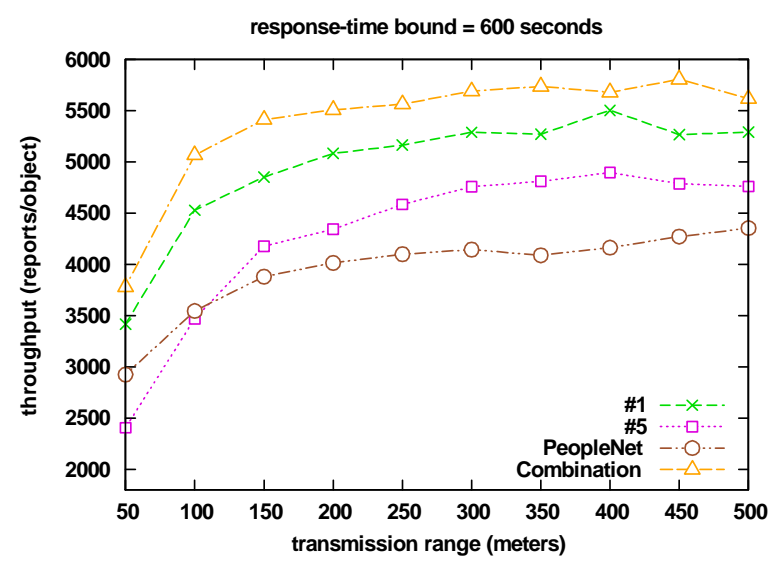

Figure. 5 Experimental results of varying transmission range

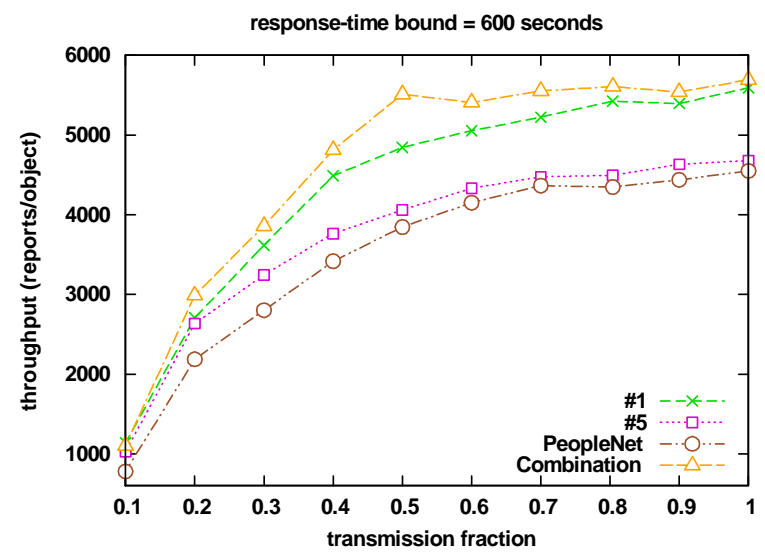

Figure. 6 Experimental results of varying transmission fraction

\section{RELEVANT WORK}

The traditional data dissemination methods can be classified into two categories, namely topology-based and location-based. Topology-based methods exploit the information about links in the network and can be further classified into flooding-based, gossiping-based, negotiationbased, and cluster-based methods. Flooding-based methods, such as [1], simply broadcast received reports to all neighbors until the destination is reached or the maximum number of hops is exceeded. Although flooding-based methods are very simple, they have inherent problems including implosion, overlap, and resource blindness, which make them neither effective nor efficient for data dissemination in mobile peer-topeer networks. Gossiping-based methods, such as [2] and [3], improve flooding-based methods by transmitting received reports to a randomly selected neighbor or the neighbors that are interested in the particular content. The advantages of gossiping-based methods include reducing the implosion and lowering the system overhead. However, the cost of determining the particular interests of each moving object can be huge and transmitting reports to a randomly selected neighbor can still cause the implosion problem and waste objects' memory, bandwidth and energy.

The negotiation-based methods, such as [4] and [5], can solve the implosion and overlap problem but they require extra data transmission which costs more memory, bandwidth, and energy. In addition, in negotiation-based methods, moving objects have to generate meta-data or a signature for every report so that negotiation can be carried out, which increases the system overhead and decreases the efficiency.

The last type of topology-based method is cluster/hierarchy-based methods, such as [6] and [7]. In cluster/ hierarchy-based methods, moving objects are grouped into clusters based on the topology of the networks and the cluster heads are randomly selected. Reports are disseminated through the network in a hierarchy or cluster manner. Specifically, reports are first disseminated to each cluster head, and then each cluster head broadcasts the reports to the member objects in its group. Cluster/hierarchy-based methods may minimize the energy consumption in moving objects in low mobility environments. However, they will fail or cost more energy in highly mobile environments since they have to maintain a hierarchy structure and frequently reselect cluster heads.

Besides topology-based methods, there are many locationbased methods which utilize the location information of moving objects to assist in data dissemination, such as [8] and [9]. In location-based methods, each moving object knows the location of itself and its neighbors through some localization techniques, such as GPS or Atomic Multilateration [9]. The simplest location-based data dissemination is Greedy Forwarding, in which each moving object transmits a report to a neighbor that is closer to the destination than itself. However, Greedy Forwarding can fail in some cases, e.g., when a report is stuck in local minima, which means that the report stays in a moving object whose neighbors are all further from the destination. Therefore, some recovery strategies are proposed, such as GPSR (Greedy Perimeter Stateless Routing [10]). Other location-based methods, such as GAF (Geographic 
Adaptive Fidelity [11]) and GEAR (Geographical and Energy Aware Routing [12]), utilize the knowledge about both location and energy to disseminate reports more efficiently. However, they only exploit the spatial information of moving objects or reports and the temporal information is ignored.

Some other methods can not be classified into any of the above two categories (topology-based and location-based). These methods include Information Fusion (e.g., [13] and [14]), Directed Diffusion (e.g., [15]), Epidemic Approach (e.g., [16]), TTDD (Two-Tier Data Dissemination [17]), PeopleNet (e.g., [18]), and 7DS (e.g., [19]). Most of these methods need some global knowledge about the network and the moving objects' states.

The method proposed in this paper also does not fall in any of the above categories, and is selective in the sense that only a subset of the reports is communicated and stored in each exchange. Selective data dissemination is still a relatively unexplored area.

\section{CONCLUSION AND FUTURE WORK}

In this paper, we addressed data dissemination in mobile peer-to-peer networks where moving objects communicate each other via short-range wireless networks and reports are disseminated through the networks in a peer-to-peer manner. We proposed a new decentralized spatio-temporal approach to the selective data dissemination. In this approach, each moving object stores and transmits a limited number of reports with the highest novelty probabilities. We defined two novelty factors, AGE and DISTANCE, to compute the novelty probability from both spatial and temporal aspects. We also proposed various strategies to compute the novelty probability by using either of the two novelty factors or their combination. A simulation system is developed to test and analyze the performance of different strategies. Experimental results showed that the combination strategy is the best one compared to other strategies and existing mobile peer-to-peer methods.

For the future work, we plan to investigate more novelty factors and use machine learning algorithms to combine them for more accurate and reasonable prediction of novelty probability. We also plan to run the simulation experiments on real mobility traces to test and analyze the properties of different novelty factors and strategies. Another direction of future research is to develop mathematical formulas for the novelty probability. Thus, the behavior of the throughput and response-time can be computed based on mathematical formulas, rather than simulations.

\section{REFERENCES}

[1] R. Oliveira, L. Bernardo, and P. Pinto, "Flooding Techniques for Resource Discovery on High Mobility MANETs", Workshop on Wireless Ad-hoc Networks, 2005.

[2] A. Datta, S. Quarteroni, and K. Aberer, "Autonomous Gossiping: A Self-Organizing Epidemic Algorithm For Selective Information Dissemination in Wireless Mobile Ad-Hoc Networks," in The International Conference on Semantics of a Networked World, 2004.

[3] Md.V. Machado, O. Goussevskaia, R.A.F. Mini, C.G. Rezende, A.A.F. Loureiro, G.R. Mateus, and J.M.S., "Data Dissemination in Autonomic Wireless Sensor Networks," IEEE Journal on Selected Areas in Communications, 23(12), pp.2305-2319, 2005.

[4] J. Kulik, W. Heinzelman, and H. Balakrishnan, "NegotiationBased Protocols for Disseminating Information in Wireless Sensor Networks," Wireless Networks, vol. 8, pp. 169-185, 2002

[5] J. Kulik, W. Rabiner, and H. Balakrishnan, "Adaptive Protocols for Information Dissemination in Wireless Sensor Networks," in The 5th Annual ACM/IEEE International Conference Mobile Computing and Networking (MOBICOM'99), pp. 174-185, 1999.

[6] K. Wang, S. A. Ayyash, and T. D. C. Little, "Attribute-Based Clustering for Information Dissemination in Wireless Sensor Networks," in The 2nd Annual IEEE Communications Society Conf. on Sensor and Ad Hoc Communications and Networks (SECON'05), Sep 2005.

[7] A. Visvanathan, J. H. Youn, and J. Deogun, "Hierarchical Data Dissemination Scheme for Large Scale Sensor Networks," in IEEE International Conference on Communications (ICC'05), pp. 3030- 3036, May 2005.

[8] H. Sabbineni and K. Chakrabarty, "Location-Aided Flooding: An Energy-Efficient Data Dissemination Protocol for Wireless Sensor Networks," IEEE Transactions on Computers, vol. 54, no. 1, pp. 36-46, Jan 2005.

[9] M. Mauve, A. Widmer, and H. Hartenstein, "A Survey on Position-Based Routing in Mobile Ad-Hoc Networks," IEEE Network, vol. 15, no. 6, pp. 30-39, 2001.

[10] B. Karp and H. T. Kung, "GPSR: Greedy Perimeter Stateless Routing for Wireless Sensor Networks," in The 6th Annual ACM/IEEE International Conference on Mobile Computing and Networking (MobiCom'00), pp. 243-254, Aug 2000.

[11] Y. Xu, J. Heidemann, and D. Estrin, "Geography-informed Energy Conservation for Ad hoc Routing," in The ACM International Conference on Mobile Computing and Networking, pp. 70-84, Rome, Italy, July 2001.

[12] Y. Yu, R. Govindan, and D. Estrin., "Geographical and Energy Aware Routing: A Recursive Data Dissemination Protocol for Wireless Sensor Networks," Technical Report UCLA/CSD-TR01-0023,UCLA, May 2001.

[13] E. F. Nakamura, C. M. S. Figueiredo, and A. A. F. Loureiro, "Information Fusion for Data Dissemination in Self-Organizing Wireless Sensor Networks," Lecture Notes in Computer Science, vol. 3420, pp. 585-593, 2005.

[14] E. F. Nakamura, F. G. Nakamura, C. M. S. Figueiredo, and A. A F. Loureiro, "Using Information Fusion to Assist Data Dissemination in Wireless Sensor Networks," Telecommunication Systems, vol.30, no.1-3, pp.237-254, 2005.

[15] C. Intanagonwiwat, R. Govindan, and D. Estrin, "Directed Diffusion: A Scalable and Robust Communication Paradigm for Sensor Networks," in The 6th Annual ACM/IEEE International Conference on Mobile Computing and Networking (MobiCom'00), Aug 2000.

[16] M. Akdere, C. C. Bilgin, O. Gerdaneri, I. Korpeoglu, O. Ulusoy, and U. Cetintemel, "A Comparison of Epidemic Algorithms in Wireless Sensor Networks," Computer Communications, vol. 29, pp. 2450-2457, 2006.

[17] H. Luo, F. Ye, J. Cheng, S. Lu, and L. Zhang, "TTDD: Two-Tier Data Dissemination in Large-Scale Wireless Sensor Networks," Wireless Networks, vol. 11, no. 1-2, pp. 161-175, Jan 2005.

[18] M. Motani, V. Srinivasan, and P. Nuggehalli, "PeopleNet: Engineering a Wireless Virtual Social Network," in Proc. MobilCom'05, Aug 2005.

[19] M. Papadopouli and H. Schulzrinne, "Effects of Power Conservation, Wireless coverage and Cooperation on Data Dissemination Among Mobile Devices," In Proc. ACM MobiHoc 2001. 